\title{
Topology of the Redefined Intuitionistic Fuzzy Rough Sets
}

\author{
Sang Min Yun D, Yeon Seok Eom D, and Seok Jong Lee
}

Department of Mathematics, Chungbuk National University, Cheongju, Korea

\section{]jfis}

\begin{abstract}
In our previous paper, we proposed a new definition of intuitionistic fuzzy rough sets. In this paper, we propose a topology for redefined intuitionistic fuzzy rough sets and investigate the basic properties of their subspaces, transition spaces, and continuous functions. Moreover, we obtain the adjointness between the categories of fuzzy rough sets and intuitionistic fuzzy rough sets. The results obtained from this new definition differ from those of previous studies.
\end{abstract}

Keywords: Fuzzy rough set, Intuitionistic fuzzy rough sets, Transition topology, Category

\section{Introduction}

Since Zadeh [1] introduced the notion of fuzzy sets, several researchers have attempted to generalize fuzzy sets using various approaches.

One approach has been to use the concept of intuitionistic fuzzy sets introduced by Atanassov [2]. Unlike fuzzy sets, an intuitionistic fuzzy set provides both a membership degree and a non-membership degree. Many important concepts in general topology have been generalized to intuitionistic fuzzy settings.

The other main approach is to utilize the concept of rough sets introduced by Pawlak [3]. The rough set theory proposes a mathematical approach to imperfect knowledge, which is expressed by the boundary region of a set. The rough set concept can be represented by topological approximations, that is, interior and closure. Zhou et al. [4]5] proposed lower and upper approximations of intuitionistic fuzzy sets. Many researchers, including us, have examined the important properties of intuitionistic fuzzy approximation operators [6-8].

Many attempts have been made with the objective of combining fuzziness and roughness.

Received: Oct. 15, 2021

Revised : Nov. 19, 2021

Accepted: Nov. 29, 2021

Correspondence to: Seok Jong Lee (sjl@cbnu.ac.kr)

๑The Korean Institute of Intelligent Systems

cCThis is an Open Access article distributed under the terms of the Creative Commons Attribution Non-Commercial License http://creativecommons.org/licenses/by-nc/ 3.0/) which permits unrestricted noncommercial use, distribution, and reproduction in any medium, provided the original work is properly cited. fuzzy rough sets. Coker [11] demonstrated that fuzzy rough sets, as proposed by Nanda and Majumdar [10], are intuitionistic L-fuzzy sets developed by Atanassov [2]. Chakrabarty et al. [12] proposed a fuzziness measure in rough sets. By combining the concepts of intuitionistic fuzzy sets and fuzzy rough sets, Samanta and Mondal [13] proposed the idea of intuitionistic fuzzy rough sets. The topology of intuitionistic fuzzy rough sets was accordingly introduced [14 15]. Bashir et al. [16] studied the topological properties of intuitionistic fuzzy rough sets under different conditions like serial, strongly serial, and left continuity.

The concept of intuitionistic fuzzy rough sets has some advantages with regard to decisionmaking. Zhan and Sun [17] discussed the rough and precision degrees of covering-based intuitionistic fuzzy rough set models. They introduced an intuitionistic fuzzy rough methodology 
to the multi-attribute decision-making problem, which is more effective than the previous models. Shanthi [18] developed a decision-making method based on the composition of intuitionistic fuzzy rough matrices on a finite universe.

In light of mathematical theory, however, the properties of the "old" intuitionistic fuzzy rough sets are extremely complicated and inadequate in terms of the extension of intuitionistic properties. Hence, remedying this flaw is critical for expanding related theories. To overcome this weakness, we introduced a new definition of fuzzy rough sets and intuitionistic fuzzy rough sets [19].

In this paper, we introduce a topology for redefined intuitionistic fuzzy rough sets and investigate the basic properties of their subspaces, transition spaces, and continuous functions. In addition, we study the categorical relation between the category of fuzzy rough sets and the category of intuitionistic fuzzy rough sets. The results obtained from this new definition are different from those obtained in previous studies. Some of our results are similar to those of Hazra et al. [15]. However, our results are consistent with the theory of intuitionistic fuzzy sets. The results help us base decision-making on a more solid foundation.

\section{New Definition of Fuzzy Rough Sets and In- tuitionistic Fuzzy Rough Sets}

In [10], the definition of fuzzy rough sets was introduced. The paper stated "We shall consider $(V, \mathfrak{B})$ to be a rough universe where $V$ is a nonempty set and $\mathfrak{B}$ is a Boolean subalgebra of the Boolean algebra of all subsets of $V$. In addition, consider a rough set. $X=\left(X_{L}, X_{U}\right) \in \mathfrak{B}^{2}$ with $X_{L} \subseteq X_{U}$. A fuzzy rough set of $X$ is an object of the form

$$
A=\left(A_{L}, A_{U}\right),
$$

where $A_{L}$ and $A_{U}$ are characterized by a pair of maps $A_{L}$ : $X_{L} \rightarrow L$ and $A_{U}: X_{U} \rightarrow L$ with $A_{L}(x) \leq A_{U}(x)$ for all $x \in X_{L}$, where $(L, \leq)$ is a fuzzy lattice."

Furthermore, the complement $\bar{A}$ of a fuzzy rough set $A=$ $\left(A_{L}, A_{U}\right)$ is defined by $(\bar{A})_{L}(x)=\left(A_{U>L}\right)^{\prime}(x), \forall x \in X_{L}$, and $(\bar{A})_{U}(x)=\left(A_{L<U}\right)^{\prime}(x), \forall x \in X_{U}$,

where $A_{U>L}(x)=A_{U}(x), \forall x \in X_{L}$ and

$$
A_{L<U}(x)= \begin{cases}A_{L}(x), & \text { if } x \in X_{L}, \\ \bigvee\left\{A_{L}(x) \mid x \in X_{L}\right\}, & \text { if } x \in X_{U}-X_{L}\end{cases}
$$

Unfortunately, if we follow this definition, the double complement of fuzzy rough set $A$ is different from $A$. This is because $X_{L}$ and $X_{U}$ could be different. The property that the double complement of a set becomes the set itself is essential in expanding related theories. To avoid this, we introduced a new definition of fuzzy rough sets and intuitionistic fuzzy rough sets by weakening the condition of the old definition. We recall some definitions and the results of our previous study.

Definition $2.1([19 \mid)$. Let $X$ be an underlying set and $(L, \leq)$ a fuzzy lattice. A fuzzy rough set of $X$ is an object of the form

$$
A=\left(A_{L}, A_{U}\right),
$$

where $A_{L}$ and $A_{U}$ are defined by a pair of maps $A_{L}: X \rightarrow L$ and $A_{U}: X \rightarrow L$ with $A_{L}(x) \leq A_{U}(x)$ for all $x \in X$. Furthermore, $\underline{0}=\left(\underline{0}_{L}, \underline{0}_{U}\right)$ is the null fuzzy rough set, and $\underline{1}=\left(\underline{1}_{L}, \underline{1}_{U}\right)$ is the entire fuzzy rough set of $X$.

The complement $\bar{A}=\left((\bar{A})_{L},(\bar{A})_{U}\right)$ of a fuzzy rough set $A=\left(A_{L}, A_{U}\right)$ of $X$ is defined by $(\bar{A})_{L}(x)=\left(A_{U}(x)\right)^{\prime}$ and $(\bar{A})_{U}(x)=\left(A_{L}(x)\right)^{\prime}$ for all $x \in X$, where $a^{\prime}$ denotes the complement for $a \in L$.

Theorem 2.2. For any fuzzy rough set $A=\left(A_{L}, A_{U}\right)$ of $X$, $\overline{(\bar{A})}=A$.

Proof. For any fuzzy rough set $A=\left(A_{L}, A_{U}\right)$ of $X$ and for any $x \in X$,

$$
(\bar{A})_{L}(x)=\left(A_{U}(x)\right)^{\prime} \leq\left(A_{L}(x)\right)^{\prime}=(\bar{A})_{U}(x) .
$$

So, $\bar{A}$ is again a fuzzy rough set. Furthermore, we have a double complement property, that is, $\overline{(\bar{A})}=A$.

Definition 2.3. [19] If $A=\left(A_{L}, A_{U}\right)$ and $B=\left(B_{L}, B_{U}\right)$ are two fuzzy rough sets of $X$ with $B \subseteq \bar{A}$, then the ordered pair $(A, B)$ is called an intuitionistic fuzzy rough set (briefly, IF rough set) of $X$. Condition $B \subseteq \bar{A}$ is called the intuitionistic condition. The complement of the IF rough set $\boldsymbol{P}=(A, B)$ of $X$ is defined by $\overline{\boldsymbol{P}}=(B, A)$.

An IF rough set is an intuitive version of a fuzzy rough set. An IF rough set has a membership degree and a nonmembership degree comprising fuzzy rough sets. Therefore, this concept is effective in dealing with systems that have a rough membership degree and rough non-membership degree.

We denote by $\operatorname{IFRS}(X)$ the collection of all IF rough sets of $X$. Usually, we use letters $X, Y, Z, \ldots$ to denote sets, letters $A, B, C, \ldots$ to denote fuzzy rough sets, and bold letters $\boldsymbol{P}, \boldsymbol{Q}, \boldsymbol{R}, \ldots$ to denote IF rough sets. 
The IF rough sets $\mathbf{0}=(\underline{0}, \underline{1})=\left(\left(\underline{0}_{L}, \underline{0}_{U}\right),\left(\underline{1}_{L}, \underline{1}_{U}\right)\right)$, and $\mathbf{1}=(\underline{1}, \underline{0})=\left(\left(\underline{1}_{L}, \underline{1}_{U}\right),\left(\underline{0}_{L}, \underline{0}_{U}\right)\right)$ are called the null IF rough set and the whole IF rough set of $X$, respectively. Clearly, $\overline{\mathbf{0}}=\mathbf{1}$ and $\overline{\mathbf{1}}=\mathbf{0}$. If we identify the crisp set $X$ with $\chi_{X}=$ $(\chi X, \underline{0})=(\underline{1}, \underline{0})=\left(\left(\underline{1}_{L}, \underline{1}_{U}\right),\left(\underline{0}_{L}, \underline{0}_{U}\right)\right)=\mathbf{1}_{X}$, then $X$ is also an IF rough set of $X$.

Definition 2.4 ([19]). Let $f: X \rightarrow Y$ be a map. Let $\boldsymbol{P}=$ $(A, B)=\left(\left(A_{L}, A_{U}\right),\left(B_{L}, B_{U}\right)\right)$ be an IF rough set of $X$. We define an image of $\boldsymbol{P}$ under $f$ as follows:

$$
f(\boldsymbol{P})=(f(A), \overline{f(\bar{B})}),
$$

where $f(A)=\left(f\left(A_{L}\right), f\left(A_{U}\right)\right)$, and $\overline{f(\bar{B})}=\left((\overline{f(\bar{B})})_{L}\right.$, $\left.(\overline{f(\bar{B})})_{U}\right)$ is defined as $(\overline{f(\bar{B})})_{L}=\overline{f\left((\bar{B})_{L}\right)}$ and $(\overline{f(\bar{B})})_{U}=$ $\overline{f\left((\bar{B})_{U}\right)}$.

Subsequently, $f(\boldsymbol{P})$ satisfies the intuitionistic condition (Remark 3.9 of [19]); hence, $f(\boldsymbol{P})$ is an IF rough set. For any IF rough set $\boldsymbol{P}=(A, B)$ of $X$ and any map $f: X \rightarrow Y$, the image $f(\boldsymbol{P})=f((A, B))$ of $\boldsymbol{P}$ under $f$ is expressed as follows: For any $y \in Y$,

$f(\boldsymbol{P})(y)=\left(\bigvee\left\{A(x) \mid x \in f^{-1}(y)\right\}, \bigwedge\left\{B(x) \mid x \in f^{-1}(y)\right\}\right)$.

Definition 2.5 ([19]). Let $f: X \rightarrow Y$ be a mapping and $\boldsymbol{Q}=(C, D)=\left(\left(C_{L}, C_{U}\right),\left(D_{L}, D_{U}\right)\right)$ be an IF rough set of $Y$. We define an inverse image of $Q$ under $f$ as:

$$
f^{-1}(\boldsymbol{Q})=\left(f^{-1}(C), f^{-1}(D)\right),
$$

where $f^{-1}(C)=\left(f^{-1}\left(C_{L}\right), f^{-1}\left(C_{U}\right)\right)$ and $f^{-1}(D)=\left(f^{-1}\left(D_{L}\right)\right.$, $\left.f^{-1}\left(D_{U}\right)\right)$.

Then, the inverse image of an IF rough set under $f$ is also an IF rough set.

For the other properties of IF rough sets, refer to [19].

\section{Categorical Properties of Intuitionistic Fuzzy Rough Sets}

One of the benefits of our new definition is the ability to construct a category of rough sets and a category of IF rough sets, where the double negation of a fuzzy rough set becomes itself. With the old definition of [15], constructing a category is difficult.

In this section, we study the connection between two concepts, that is, IF rough sets and fuzzy rough sets.
Definition 3.1. Let $A$ be a fuzzy rough set of $X$ and $C$ a fuzzy rough set of $Y$. A function $f: X \rightarrow Y$ is called a function from $A$ to $C$, denoted by $f: A \rightarrow C$, if $f(A) \subseteq C$.

Definition 3.2. Let $\boldsymbol{P}=(A, B)$ be an IF rough set of $X$ and $\boldsymbol{Q}=(C, D)$ an IF rough set of $Y$. A function $f: X \rightarrow Y$ is called a function from $\boldsymbol{P}$ to $\boldsymbol{Q}$, denoted by $f: \boldsymbol{P} \rightarrow \boldsymbol{Q}$, if $f(\boldsymbol{P}) \subseteq \boldsymbol{Q}$.

Now, we are ready to define functors between two categories. Let FRS be the category of all fuzzy rough sets and functions on fuzzy rough sets. Let IFRS is the category of all IF rough sets and functions on IF rough sets.

Theorem 3.3. Define $F_{1}:$ IFRS $\rightarrow$ FRS by

$$
\begin{aligned}
& F_{1}(\boldsymbol{P})=F_{1}((A, B))=A, \\
& F_{1}(f)=f .
\end{aligned}
$$

Then $F_{1}$ is a functor from IFRS to FRS.

Proof. Clearly, $F_{1}(\boldsymbol{P})=A$ is a fuzzy rough set of $X$ for any IF rough set $\boldsymbol{P}=(A, B)$ of $X$. Let $f: \boldsymbol{P} \rightarrow \boldsymbol{Q}$ be a function from the IF rough set $\boldsymbol{P}=(A, B)=\left(\left(A_{L}, A_{U}\right),\left(B_{L}, B_{U}\right)\right)$ of $X$ to an IF rough set $\boldsymbol{Q}=(C, D)=\left(\left(C_{L}, C_{U}\right),\left(D_{L}, D_{U}\right)\right)$ of $Y$. Then, $f$ is a function $f: X \rightarrow Y$ such that $f(\boldsymbol{P}) \subseteq \boldsymbol{Q}$. Thus, $F_{1}(f)=f$ is a function $f: X \rightarrow Y$ such that $f(A) \subseteq C$. Thus, $f: A \rightarrow C$ is a function of the fuzzy rough sets. Hence $F_{1}$ is a functor from IFRS to FRS.

Similarly, we have the following functor.

Theorem 3.4. Define $F_{2}:$ IFRS $\rightarrow$ FRS by

$$
F_{2}(\boldsymbol{P})=F_{2}((A, B))=B, \quad F_{2}(f)=f .
$$

Then, $F_{2}$ is a functor from IFRS to FRS.

Theorem 3.5. Define $G_{1}:$ FRS $\rightarrow$ IFRS by

$$
G_{1}(A)=(A, \bar{A}), \quad G_{1}(f)=f .
$$

Then, $G_{1}$ is a functor from FRS to IFRS.

Proof. Clearly, $G_{1}(A)=(A, \bar{A})$ is an IF rough set of $X$ for any fuzzy rough set $A$ of $X$. Let $f: A \rightarrow C$ be a function from a fuzzy rough set $A$ of $X$ to a fuzzy rough set $C$ of $Y$. Then, $f$ is a function $f: X \rightarrow Y$ such that $f(A) \subseteq C$. Thus $f((A, \bar{A}))=$ $(f(A), \overline{f(\overline{\bar{A}})})=(f(A), \overline{f(A)}) \subseteq(C, \bar{C})$. Therefore, $G_{1}(f)=$ $f$ is a function $f: X \rightarrow Y$ such that $f((A, \bar{A})) \subseteq(C, \bar{C})$, that is, $f:(A, \bar{A})) \rightarrow(C, \bar{C})$ is a function of IF rough sets. Hence, $G_{1}$ is a functor from FRS to IFRS. 
Similarly, we have the following functor.

Theorem 3.6. Define $G_{2}:$ FRS $\rightarrow$ IFRS by

$$
G_{2}(A)=(\bar{A}, A), G_{2}(f)=f .
$$

Then, $G_{2}$ is a functor from FRS to IFRS.

As in the following two theorems, we have two adjointnesses between the above functors.

Theorem 3.7. The functor $F_{1}:$ IFRS $\rightarrow$ FRS is a right adjoint of functor $G_{1}:$ FRS $\rightarrow$ IFRS.

Proof. The following diagram commutes.

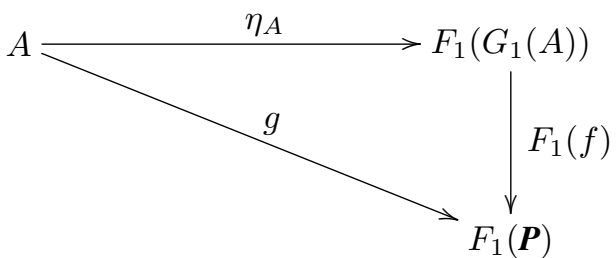

Take $A$ in FRS. Then, $G_{1}(A)=(A, \bar{A})$ is a member of IFRS. Take any $\boldsymbol{P}=(C, D)$ in IFRS and any function $g: A \rightarrow$ $F_{1}(\boldsymbol{P})=C$. Then, $g: X \rightarrow Y$ is a function such that $g(A) \subseteq$ $C$. By the intuitionistic condition, we have $C \subseteq \bar{D}$. Hence, $g(A) \subseteq \bar{D}$, i.e., $\overline{g(A)} \supseteq D$. Thus, $(g(A), \overline{g(A)}) \subseteq(C, D)$. Thus, we have $g((A, \bar{A}))=(g(A), g(\overline{\bar{A}}))=(g(A), \overline{g(A)}) \subseteq$ $\boldsymbol{P}$. Hence, there exists a unique $f=g: G_{1}(A) \rightarrow \boldsymbol{P}$ and $\eta_{A}: A \rightarrow F_{1}\left(G_{1}(A)\right)$ such that $F_{1}(f) \circ \eta_{A}=g$. Hence, $F_{1}:$ IFRS $\rightarrow$ FRS is a right adjoint of $G_{1}:$ FRS $\rightarrow$ IFRS.

Theorem 3.8. The functor $F_{2}:$ IFRS $\rightarrow$ FRS is a right adjoint of functor $G_{2}:$ FRS $\rightarrow$ IFRS.

Proof. Similar to the above proof.

We then obtained a categorical relation between the category of fuzzy rough sets and the category of IF rough sets.

\section{Topology of Intuitionistic Fuzzy Rough Sets}

A basic approach for studying the application of a new mathematical system is topology because the topological structure explains the convergence and neighborhoodness of a system. In this section, we introduce the topology of IF rough sets.

Definition 4.1. Let $X$ be a nonempty set and $\mathcal{T}$ be a family of IF rough sets of $X$ such that
(1) $\mathbf{0}, \mathbf{1}_{X} \in \mathcal{T}$,

(2) $\boldsymbol{P} \cap \boldsymbol{Q} \in \mathcal{T}$ for all $\boldsymbol{P}, \boldsymbol{Q} \in \mathcal{T}$,

(3) $\boldsymbol{P}_{i} \in \mathcal{T}$ for all $i \in \Delta$ implies $\bigcup_{i \in \Delta} \boldsymbol{P}_{i} \in \mathcal{T}$.

Then, $\mathcal{T}$ is called a topology of IF rough sets of $X$, and the pair $(X, \mathcal{T})$ is called a topological space of IF rough sets of $X$. Every member of $\mathcal{T}$ is called an open IF rough set. An IF rough set $\boldsymbol{Q}$ is said to be closed if $\bar{Q} \in \mathcal{T}$. Let $\mathcal{F}$ denote the collection of all closed IF rough sets of $(X, \mathcal{T})$. If $\mathcal{T}=\{\mathbf{0}, \mathbf{1}\}$, then $\mathcal{T}$ is a topology of IF rough sets of $X$. This topology is called a indiscrete topology. The discrete topology of IF rough sets of $X$ contains all IF rough sets of $X$.

Theorem 4.2. The collection of $\mathcal{F}$ of all closed IF rough sets of $(X, \mathcal{T})$ satisfies the following properties:

(1) $\mathbf{0}, \mathbf{1}_{X} \in \mathcal{F}$

(2) $\boldsymbol{P} \cup \boldsymbol{Q} \in \mathcal{F}$, for all $\boldsymbol{P}, \boldsymbol{Q} \in \mathcal{F}$.

(3) $\boldsymbol{P}_{i} \in \mathcal{F}, i \in \Delta$ implies $\bigcap_{i \in \Delta} \boldsymbol{P}_{i} \in \mathcal{F}$.

Proof. Obvious from Definition 4.1 .

Definition 4.3. Let $\boldsymbol{P}$ be an IF rough set of $X$. The interior of $\boldsymbol{P}$ in $(X, \mathcal{T})$ is the union of all open IF rough sets in $(X, \mathcal{T})$ contained in $\boldsymbol{P}$ and is denoted by int $\mathcal{T} \boldsymbol{P}$.

Definition 4.4. Let $\boldsymbol{P}$ be an IF rough set of $X$. The closure of $\boldsymbol{P}$ in $(X, \mathcal{T})$ is the intersection of all closed IF rough sets in $(X, \mathcal{T})$ containing $\boldsymbol{P}$ and is denoted by $\mathrm{cl}_{\mathcal{T}} \boldsymbol{P}$.

$\operatorname{cl}_{\mathcal{T}} \boldsymbol{P}$ is the smallest closed IF rough set containing $\boldsymbol{P}$, and $\boldsymbol{P}$ is closed if and only if $\boldsymbol{P}=\operatorname{cl}_{\mathcal{T}} \boldsymbol{P}$. In addition, $\operatorname{int}_{\mathcal{T}} \boldsymbol{P}$ is the largest open IF rough set contained in $\boldsymbol{P}$, and $\boldsymbol{P}$ is open if and only if $\boldsymbol{P}=\operatorname{int}_{\mathcal{T}} \boldsymbol{P}$. We have the following properties directly from the definitions.

Theorem 4.5. (1) $\mathrm{cl}_{\mathcal{T}} \mathbf{0}=\mathbf{0} \in \mathcal{F}$.

(2) $\operatorname{cl}_{\mathcal{T}}\left(\operatorname{cl}_{\mathcal{T}} \boldsymbol{P}\right)=\operatorname{cl}_{\mathcal{T}} \boldsymbol{P}$.

(3) $\boldsymbol{P} \subseteq \boldsymbol{Q}$ implies $\operatorname{cl}_{\mathcal{T}} \boldsymbol{P} \subseteq \operatorname{cl}_{\mathcal{T}} \boldsymbol{Q}$.

(4) $\operatorname{cl}_{\mathcal{T}}(\boldsymbol{P} \cup \boldsymbol{Q})=\operatorname{cl}_{\mathcal{T}} \boldsymbol{P} \cup \mathrm{cl}_{\mathcal{T}} \boldsymbol{Q}$

(5) $\operatorname{cl}_{\mathcal{T}}(\boldsymbol{P} \cap \boldsymbol{Q}) \subseteq \operatorname{cl}_{\mathcal{T}} \boldsymbol{P} \cap \mathrm{cl}_{\mathcal{T}} \boldsymbol{Q}$.

Definition 4.6. Let $(X, \mathcal{T})$ and $(Y, \mathcal{U})$ be two topological spaces of the IF rough sets. And let $f: X \rightarrow Y$ be a mapping. Then, $f:(X, \mathcal{T}) \rightarrow(Y, \mathcal{U})$ is said to be IFR continuous if $f^{-1}(\boldsymbol{Q}) \in \mathcal{T}$ for all $\boldsymbol{Q} \in \mathcal{U}$. 
Let $(X, \mathcal{T}),(Y, \mathcal{U})$, and $(Z, \mathcal{V})$ be the topological spaces of the IF rough sets. If $f:(X, \mathcal{T}) \rightarrow(Y, \mathcal{U})$, and $g:(Y, \mathcal{U}) \rightarrow$ $(Z, \mathcal{V})$ are IFR continuous, then $g \circ f:(X, \mathcal{T}) \rightarrow(Z, \mathcal{V})$ is clearly IFR continuous.

The continuous function is characterized by closed sets and closure as follows.

Theorem 4.7. The following statements are equivalent:

(1) $f:(X, \mathcal{T}) \rightarrow(Y, \mathcal{U})$ is IFR continuous.

(2) $f^{-1}(\boldsymbol{Q})$ is closed IF rough set in $(X, \mathcal{T})$, for any closed IF rough set $\boldsymbol{Q}$ in $(Y, \mathcal{U})$.

(3) $f\left(\operatorname{cl}_{\mathcal{T}} \boldsymbol{P}\right) \subseteq \operatorname{cl}_{\mathcal{U}}(f(\boldsymbol{P}))$, for any IF rough set $\boldsymbol{P}$ in $(X, \mathcal{T})$.

Proof. (1) $\Rightarrow$ (2) Let $f$ be an IFR continuous function and $Q$ be a closed IF rough set in $(Y, \mathcal{U})$. Then, $\overline{\boldsymbol{Q}}$ is an open IF rough set in $(Y, \mathcal{U})$. As $f$ is IFR continuous, $f^{-1}(\overline{\boldsymbol{Q}})=\overline{f^{-1}(\boldsymbol{Q})}$ is an open IF rough set in $(X, \mathcal{T})$. Thus, $f^{-1}(\boldsymbol{Q})$ is closed in $(X, \mathcal{T})$.

(2) $\Rightarrow$ (3) Let $\boldsymbol{P}$ be an IF rough set of $X$. Because $\operatorname{cl}_{\mathcal{U}}(f(\boldsymbol{P}))$ is closed in $(Y, \mathcal{U})$, by $(2), f^{-1}\left(\operatorname{cl}_{\mathcal{U}}(f(\boldsymbol{P}))\right)$ is closed in $(X, \mathcal{T})$. Because $\boldsymbol{P} \subseteq f^{-1}\left(\operatorname{cl}_{\mathcal{U}}(f(\boldsymbol{P}))\right)$, we have $\operatorname{cl}_{\mathcal{T}} \boldsymbol{P} \subseteq f^{-1}\left(\operatorname{cl}_{\mathcal{U}}(f(\boldsymbol{P}))\right)$. Therefore $f\left(\operatorname{cl}_{\mathcal{T}} \boldsymbol{P}\right) \subseteq f\left(f^{-1}\left(\operatorname{cl}_{\mathcal{U}}(f(\boldsymbol{P}))\right)\right) \subseteq \operatorname{cl}_{\mathcal{U}}(f(\boldsymbol{P}))$.

(3) $\Rightarrow$ (1) Let $\boldsymbol{Q}$ be an open IF rough set of $Y$. Because $\overline{\boldsymbol{Q}}$ is closed in $Y, \overline{\boldsymbol{Q}}=\operatorname{cl}_{\mathcal{U}} \overline{\boldsymbol{Q}}$. Thus, we have $f\left(\operatorname{cl}_{\mathcal{T}}\left(f^{-1}(\overline{\boldsymbol{Q}})\right) \subseteq\right.$ $\operatorname{cl}_{\mathcal{U}}\left(f\left(f^{-1}(\overline{\boldsymbol{Q}})\right)\right) \subseteq \operatorname{cl}_{\mathcal{U}} \overline{\boldsymbol{Q}}=\overline{\boldsymbol{Q}}$. Therefore, $\operatorname{cl}_{\mathcal{T}}\left(f^{-1}(\overline{\boldsymbol{Q}})\right) \subseteq$ $f^{-1}(\overline{\boldsymbol{Q}})$. Hence, $\operatorname{cl}_{\mathcal{T}}\left(f^{-1}(\overline{\boldsymbol{Q}})\right)=f^{-1}(\overline{\boldsymbol{Q}})$. Because $f^{-1}(\overline{\boldsymbol{Q}})=$ $\overline{f^{-1}(\boldsymbol{Q})}$, we have that $f^{-1}(\boldsymbol{Q})$ is open in $X$. Hence $f:(X, \mathcal{T})$ $\rightarrow(Y, \mathcal{U})$ is IFR continuous.

\section{Subspace Topology}

Let $Y$ be a crisp subset of $X$. We denote the IF rough set $\left.\left(\chi_{Y}, 1-\chi_{Y}\right)=\left(\left(\left(\chi_{Y}\right)_{L},\left(\chi_{Y}\right)_{U}\right),\left(1-\chi_{Y}\right)_{L},\left(1-\chi_{Y}\right)_{U}\right)\right)$ by $\chi_{Y}$ or $\mathbf{1}_{Y}$, and the IF rough set $\overline{\chi_{Y}}=\left(1-\chi_{Y}, \chi_{Y}\right)=((1-$ $\left.\left.\left.\chi_{Y}\right)_{L},\left(1-\chi_{Y}\right)_{U}\right),\left(\left(\chi_{Y}\right)_{L},\left(\chi_{Y}\right)_{U}\right)\right)$ by $\mathbf{0}_{Y}$. If we take $Y=$ $X$, then $\mathbf{1}_{Y}=\mathbf{1}$ and $\mathbf{0}_{Y}=\mathbf{0}$. Clearly, $\mathbf{0}_{Y}$ is a complement of $\mathbf{1}_{Y}$ and vice versa, as an IF rough set.

Theorem 5.1. Let $(X, \mathcal{T})$ be a topological space of IF rough sets of $X$. Let $Y$ be a crisp subset of $X$. Then, the family $\mathcal{T}_{Y}=\left\{\boldsymbol{P} \cap \mathbf{1}_{Y} \mid \boldsymbol{P} \in \mathcal{T}\right\}$ of IF rough sets of $Y$ satisfies the axioms of Definition 4.1.

This topology is called the subspace topology of $(X, \mathcal{T})$ on $Y$. Every member of $\mathcal{T}_{Y}$ is called an open IF rough set of $\left(Y, \mathcal{T}_{Y}\right)$. Clearly, $\mathbf{0} \in \mathcal{T}_{Y}$ and $\mathbf{1}_{Y} \in \mathcal{T}_{Y}$.
If $\boldsymbol{Q} \in \mathcal{T}_{Y}$, then $\overline{\boldsymbol{Q}}_{Y}$ is called a closed IF rough set of $\left(Y, \mathcal{T}_{Y}\right)$, where $\overline{\boldsymbol{Q}}_{Y}=\overline{\boldsymbol{Q}} \cap \mathbf{1}_{Y}$. Let

$$
\mathcal{F}_{Y}=\left\{\overline{\boldsymbol{Q}}_{Y}=\overline{\boldsymbol{Q}} \cap \mathbf{1}_{Y} \mid \boldsymbol{Q} \in \mathcal{T}_{Y}\right\} .
$$

Then, $\mathcal{F}_{Y}$ is closed under arbitrary intersections and finite unions. Since $\mathbf{1}_{Y} \in \mathcal{T}_{Y}$, we have $\mathbf{0}=\overline{\mathbf{1}_{Y}} \cap \mathbf{1}_{Y} \in \mathcal{F}_{Y}$. In addition, $\mathbf{1}_{Y}=\overline{\mathbf{0}} \cap \mathbf{1}_{Y} \in \mathcal{F}_{Y}$.

Theorem 5.2. Let $(X, \mathcal{T})$ be a topological space of IF rough sets of $X$. Let $\boldsymbol{P}$ be an IF rough set of $Y$ with $Y \subseteq X$. Then $\operatorname{cl}_{Y}(\boldsymbol{P})=\operatorname{cl}_{X}(\boldsymbol{P}) \cap \mathbf{1}_{Y}$.

Proof. Suppose that $\left\{\boldsymbol{F}_{i} \mid i \in J\right\}$ is the collection of all closed IF rough sets of $Y$ that contain $\boldsymbol{P}$. For each $i$, we can choose an open IF rough set $\boldsymbol{T}_{i}$ of $X$ such that $\boldsymbol{F}_{i}=\overline{\boldsymbol{T}_{i}} \cap \mathbf{1}_{Y}$. Thus

$$
\begin{aligned}
\operatorname{cl}_{Y}(\boldsymbol{P}) & =\bigcap_{i \in J} \boldsymbol{F}_{i}=\bigcap_{i \in J}\left(\overline{\boldsymbol{T}_{i}} \cap \mathbf{1}_{Y}\right)=\left(\bigcap_{i \in J} \overline{\boldsymbol{T}_{i}}\right) \cap \mathbf{1}_{Y} \\
& \supseteq \bigcap\{\boldsymbol{Q} \mid \boldsymbol{Q} \text { is a closed IF rough } \\
& \quad \text { set of } X \text { and } \boldsymbol{Q} \supseteq \boldsymbol{P}\} \cap \mathbf{1}_{Y} \\
& =\operatorname{cl}_{X}(\boldsymbol{P}) \cap \mathbf{1}_{Y} .
\end{aligned}
$$

Moreover, $\mathbf{1}_{Y} \cap \mathrm{cl}_{X}(\boldsymbol{P})$ is a closed IF rough set of $Y$ that contains $\boldsymbol{P}$. Thus, $\operatorname{cl}_{Y}(\boldsymbol{P}) \subseteq \operatorname{cl}_{X}(\boldsymbol{P}) \cap \mathbf{1}_{Y}$. Hence $\operatorname{cl}_{Y}(\boldsymbol{P})=$ $\operatorname{cl}_{X}(\boldsymbol{P}) \cap \mathbf{1}_{Y}$.

Theorem 5.3. If $f:(X, \mathcal{T}) \rightarrow(Y, \mathcal{U})$ is continuous and $Z \subseteq$ $X$, then $f:\left(Z, \mathcal{T}_{Z}\right) \rightarrow(Y, \mathcal{U})$ is continuous.

Proof. For any open IF rough set $\boldsymbol{Q}$ of $Y,\left(\left.f\right|_{Z}\right)^{-1}(\boldsymbol{Q})=\chi_{Z} \cap$ $f^{-1}(\boldsymbol{Q})$. Because $f^{-1}(\boldsymbol{Q}) \in \mathcal{T}$, we have $\left(\left.f\right|_{Z}\right)^{-1}(\boldsymbol{Q}) \in \mathcal{T}_{Z}$ Hence, $f:\left(Z, \mathcal{T}_{Z}\right) \rightarrow(Y, \mathcal{U})$ is continuous.

Theorem 5.4. If $f:(X, \mathcal{T}) \rightarrow(Y, \mathcal{U})$ is continuous, then $f:(X, \mathcal{T}) \rightarrow\left(f(X), \mathcal{U}_{f(X)}\right)$ is continuous.

Proof. Take $\boldsymbol{Q} \in \mathcal{U}_{f(X)}$. Then, $\boldsymbol{Q}=\chi_{f(X)} \cap \boldsymbol{Q}^{\prime}$ for some $\boldsymbol{Q}^{\prime} \in \mathcal{U}$. Thus,

$$
\begin{aligned}
f^{-1}(\boldsymbol{Q}) & =f^{-1}\left(\chi_{f(X)}\right) \cap f^{-1}\left(\boldsymbol{Q}^{\prime}\right) \\
& =\chi_{X} \cap f^{-1}\left(\boldsymbol{Q}^{\prime}\right) \\
& =f^{-1}\left(\boldsymbol{Q}^{\prime}\right) \in \mathcal{T}
\end{aligned}
$$

Hence, $f:(X, \mathcal{T}) \rightarrow\left(f(X), \mathcal{U}_{f(X)}\right)$ is continuous.

\section{Transition Topology}

Hazra et al. [15] defined the subspace topology, but it is, in fact, a new concept different from the subspace topology. Conse- 
quently, we suggest a new terminology "transition topology." Insofar as we take the characteristic function of a crisp set, the transition topology and the subspace topology are the same.

Definition 6.1. Let $\boldsymbol{P}$ be an IF rough set. A topology on $\boldsymbol{P}$ is a collection $\mathcal{T}$ of IF rough subsets of $\boldsymbol{P}$ such that the following are true:

(1) $\mathbf{0}, \boldsymbol{P} \in \mathcal{T}$.

(2) If $\boldsymbol{P}_{1}, \boldsymbol{P}_{2} \in \mathcal{T}$, then $\boldsymbol{P}_{1} \cap \boldsymbol{P}_{2} \in \mathcal{T}$.

(3) If $\boldsymbol{P}_{i} \in \mathcal{T}$ for all $i \in \Delta$, then $\bigcup_{i \in \Delta} \boldsymbol{P}_{i} \in \mathcal{T}$.

Theorem 6.2. Let $\mathcal{T}$ be a topology of IF rough sets of $X$ and $\boldsymbol{P} \in \operatorname{IFRS}(X)$. Then, $\mathcal{T}_{\boldsymbol{P}}=\{\boldsymbol{P} \cap \boldsymbol{R} \mid \boldsymbol{R} \in \mathcal{T}\}$ is clearly a topology on $\boldsymbol{P}$, which is called a transition topology on $\boldsymbol{P}$ of $(X, \mathcal{T})$. The pair $\left(\boldsymbol{P}, \mathcal{T}_{\boldsymbol{P}}\right)$ is called a transition space of $(X, \mathcal{T})$.

If we take $\boldsymbol{P}=\chi_{E}$ for some crisp subset $E$ of $X$, then the transition topology $\mathcal{T}_{\boldsymbol{P}}$ becomes the subspace topology on $E$ of $(X, \mathcal{T})$

Every member of $\mathcal{T}_{\boldsymbol{P}}$ is called an open IF rough set of $\left(\boldsymbol{P}, \mathcal{T}_{\boldsymbol{P}}\right)$. If $\boldsymbol{Q} \in \mathcal{T}_{\boldsymbol{P}}$, then $\overline{\boldsymbol{Q}}_{\boldsymbol{P}}$ is called a closed IF rough set of $\left(\boldsymbol{P}, \mathcal{T}_{\boldsymbol{P}}\right)$, where $\overline{\boldsymbol{Q}}_{\boldsymbol{P}}=\boldsymbol{P} \cap \overline{\boldsymbol{Q}}$. Let

$$
\mathcal{F}_{\boldsymbol{P}}=\left\{\overline{\boldsymbol{Q}}_{\boldsymbol{P}}=\boldsymbol{P} \cap \overline{\boldsymbol{Q}} \mid \boldsymbol{Q} \in \mathcal{T}_{\boldsymbol{P}}\right\} \cup\{\mathbf{0}\} .
$$

Subsequently, $\mathcal{F}_{\boldsymbol{P}}$ is a family of closed IF rough sets in $\left(\boldsymbol{P}, \mathcal{T}_{\boldsymbol{P}}\right)$.

Theorem 6.3. $\mathcal{F}_{\boldsymbol{P}}$ is closed under arbitrary intersections and finite unions.

Proof. Obvious from Definition 6.1.

Remark 6.4. Let $\boldsymbol{S} \in \mathcal{T}_{\boldsymbol{P}}$. Then, $\overline{\boldsymbol{S}}_{\boldsymbol{P}}=\boldsymbol{P} \cap \overline{\boldsymbol{S}} \in \mathcal{F}_{\boldsymbol{P}}$. Now,

$$
\begin{aligned}
{\overline{\left(\overline{\boldsymbol{S}}_{\boldsymbol{P}}\right)_{\boldsymbol{P}}}} & =\boldsymbol{P} \cap \overline{\left(\overline{\boldsymbol{S}}_{\boldsymbol{P}}\right)}=\boldsymbol{P} \cap \overline{\boldsymbol{P} \cap \overline{\boldsymbol{S}}}=\boldsymbol{P} \cap(\overline{\boldsymbol{P}} \cup \boldsymbol{S}) \\
& =(\boldsymbol{P} \cap \overline{\boldsymbol{P}}) \cup \boldsymbol{S},
\end{aligned}
$$

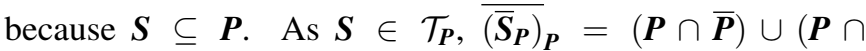
$\boldsymbol{R})$ for some $\boldsymbol{R} \in \mathcal{T}$. Thus, ${\overline{\left(\overline{\boldsymbol{S}_{\boldsymbol{P}}}\right)_{\boldsymbol{P}}}}=\boldsymbol{P} \cap(\overline{\boldsymbol{P}} \cup \boldsymbol{R})$. Hence, $\overline{\left(\bar{S}_{P}\right)_{P}}$ does not necessarily belong to $\mathcal{T}_{P}$. However,

$$
\begin{aligned}
& \overline{\left({\overline{\left(\overline{S_{P}}\right)_{P}}}_{\boldsymbol{P}}\right.}=\boldsymbol{P} \cap \overline{\left(\overline{\left.\left(\overline{\boldsymbol{S}}_{\boldsymbol{P}}\right)_{\boldsymbol{P}}\right)}\right.}=\boldsymbol{P} \cap \overline{((\boldsymbol{P} \cap \overline{\boldsymbol{P}}) \cup \boldsymbol{S})}, \\
& =\boldsymbol{P} \cap(\overline{(\boldsymbol{P} \cap \overline{\boldsymbol{P}})} \cap \overline{\boldsymbol{S}})=\boldsymbol{P} \cap((\overline{\boldsymbol{P}} \cup \boldsymbol{P}) \cap \overline{\boldsymbol{S}}) \\
& =(\boldsymbol{P} \cap(\overline{\boldsymbol{P}} \cup \boldsymbol{P})) \cap \overline{\boldsymbol{S}}=\boldsymbol{P} \cap \overline{\boldsymbol{S}}=\overline{\boldsymbol{S}}_{\boldsymbol{P}} \in \mathcal{F}_{\boldsymbol{P}} .
\end{aligned}
$$

By the above remark, we can give the following definition.
Definition 6.5. The collection

$$
\mathcal{T}_{\overline{\boldsymbol{P}}}=\left\{{\overline{\left(\overline{\boldsymbol{S}}_{\boldsymbol{P}}\right)_{\boldsymbol{P}}}}=(\boldsymbol{P} \cap \overline{\boldsymbol{P}}) \cup \boldsymbol{S} \mid \boldsymbol{S} \in \mathcal{T}_{\boldsymbol{P}}\right\} \cup\{\mathbf{0}\},
$$

forms a topology of IF rough sets on $\boldsymbol{P}$. This is called the second transition topology of $(X, \mathcal{T})$ on $\boldsymbol{P}$. The pair $\left(\boldsymbol{P}, \mathcal{T}_{\bar{P}}\right)$ is called the second transition space of $(X, \mathcal{T})$.

If we take $\boldsymbol{P}=\chi_{E}$ for some crisp subset $E$ of $X$, then the second transition topology $\mathcal{T}_{\bar{P}}$ becomes the subspace topology on $E$ of $(X, \mathcal{T})$.

$\mathcal{F}_{\boldsymbol{P}}$ is also a family of closed IF rough sets in $\left(\boldsymbol{P}, \mathcal{T}_{\overline{\boldsymbol{P}}}\right)$. Thus, there exist two topologies of IF rough sets on $\boldsymbol{P}$. $\mathcal{T}_{\boldsymbol{P}}$ is the transition topology of $(X, \mathcal{T})$ on $\boldsymbol{P}$, and $\mathcal{T}_{\bar{P}}$ is another transition topology of $(X, \mathcal{T})$ on $\boldsymbol{P}$. We briefly refer to $\mathcal{T}_{\boldsymbol{P}}$ and $\mathcal{T}_{\bar{P}}$ first and second topologies, respectively, on $\boldsymbol{P}$ if there is no confusion about the topological space $(X, \mathcal{T})$ of IF rough sets.

Remark 6.6. In general, $\mathcal{T}_{\boldsymbol{P}}$ and $\mathcal{T}_{\bar{P}}$ are different. For $\boldsymbol{P} \in$ IFRS $(X)$, let $\mathcal{T}_{\boldsymbol{P}}$ and $\mathcal{T}_{\bar{P}}$ be the first and second topologies on $\boldsymbol{P}$ that is, $\mathcal{T}_{\boldsymbol{P}}=\{\boldsymbol{P} \cap \boldsymbol{R} \mid \boldsymbol{R} \in \mathcal{T}\}$, and

$$
\begin{aligned}
\mathcal{T}_{\overline{\boldsymbol{P}}} & =\left\{(\boldsymbol{P} \cap \overline{\boldsymbol{P}}) \cup \boldsymbol{S} \mid \boldsymbol{S} \in \mathcal{T}_{\boldsymbol{P}}\right\} \cup\{\mathbf{0}\}, \\
& =\{\boldsymbol{P} \cap(\overline{\boldsymbol{P}} \cup \boldsymbol{R}) \mid \boldsymbol{R} \in \mathcal{T}\} \cup\{\mathbf{0}\} .
\end{aligned}
$$

If $\mathcal{T}$ is an indiscrete topology of IF rough sets of $X$, then $\mathcal{T}_{\boldsymbol{P}}=\{\boldsymbol{P}, \mathbf{0}\}$ and $\mathcal{T}_{\overline{\boldsymbol{P}}}=\{\boldsymbol{P}, \mathbf{0}, \boldsymbol{P} \cap \overline{\boldsymbol{P}}\}$. Thus, $\mathcal{T}_{\boldsymbol{P}} \varsubsetneqq \mathcal{T}_{\overline{\boldsymbol{P}}}$.

If $\mathcal{T}$ is a discrete topology, then $\mathcal{T}_{\boldsymbol{P}}=\{\boldsymbol{S} \in \operatorname{IFRS}(X) \mid \boldsymbol{S} \subseteq$ $\boldsymbol{P}\}, \mathcal{T}_{\overline{\boldsymbol{P}}}=\{\boldsymbol{S} \in \operatorname{IFRS}(X) \mid \boldsymbol{P} \cap \overline{\boldsymbol{P}} \subseteq \boldsymbol{S} \subseteq \boldsymbol{P}\} \cup\{\mathbf{0}\}$. Thus, $\mathcal{T}_{\bar{P}} \varsubsetneqq \mathcal{T}_{P}$.

Remark 6.7. Let $\boldsymbol{R} \in \operatorname{IFRS}(X)$ such that $\boldsymbol{R} \subseteq \boldsymbol{P}$. The closures of $\boldsymbol{R}$ in $\left(\boldsymbol{P}, \mathcal{T}_{\boldsymbol{P}}\right)$ and $\left(\boldsymbol{P}, \mathcal{T}_{\overline{\boldsymbol{P}}}\right)$ are denoted by $\mathrm{cl}_{\mathcal{T}_{\boldsymbol{P}}} \boldsymbol{R}$ and $\mathrm{cl}_{\mathcal{T}_{\bar{P}}} \boldsymbol{R}$, respectively. Because the closed IF rough sets in $\left(\boldsymbol{P}, \mathcal{T}_{\boldsymbol{P}}\right)$ and $\left(\boldsymbol{P}, \mathcal{T}_{\overline{\boldsymbol{P}}}\right)$ are the same, $\mathrm{cl}_{\mathcal{T}_{\boldsymbol{P}}} \boldsymbol{R}=\mathrm{cl}_{\mathcal{T}_{\bar{P}}} \boldsymbol{R}$. Furthermore,

$$
\begin{aligned}
\operatorname{cl}_{\mathcal{T}_{\boldsymbol{P}}} \boldsymbol{R} & =\bigcap\left\{\overline{\boldsymbol{G}}_{\boldsymbol{P}} \mid \overline{\boldsymbol{G}}_{\boldsymbol{P}} \supseteq \boldsymbol{R} ; \boldsymbol{G} \in \mathcal{T}_{\boldsymbol{P}}\right\} \\
& =\bigcap\left\{\boldsymbol{P} \cap \overline{\boldsymbol{G}} \mid \boldsymbol{P} \cap \overline{\boldsymbol{G}} \supseteq \boldsymbol{R} ; \boldsymbol{G} \in \mathcal{T}_{\boldsymbol{P}}\right\} \\
& =\boldsymbol{P} \cap\left(\bigcap\left\{\overline{\boldsymbol{G}} \mid \overline{\boldsymbol{G}} \supseteq \boldsymbol{R} ; \boldsymbol{G} \in \mathcal{T}_{\boldsymbol{P}}\right\}\right) \\
& =\boldsymbol{P} \cap(\bigcap\{\overline{(\boldsymbol{P} \cap \boldsymbol{Q})} \mid \overline{(\boldsymbol{P} \cap \boldsymbol{Q})} \supseteq \boldsymbol{R} ; \boldsymbol{Q} \in \mathcal{T}\}) \\
& \subseteq \boldsymbol{P} \cap(\overline{\boldsymbol{P}} \cup(\bigcap\{\overline{\boldsymbol{Q}} \mid \overline{\boldsymbol{Q}} \supseteq \boldsymbol{R} ; \boldsymbol{Q} \in \mathcal{T}\})) \\
& =\boldsymbol{P} \cap\left(\overline{\boldsymbol{P}} \cup \mathrm{cl}_{\mathcal{T}} \boldsymbol{R}\right) \\
& =(\boldsymbol{P} \cap \overline{\boldsymbol{P}}) \cup\left(\boldsymbol{P} \cap \mathrm{cl}_{\mathcal{T}} \boldsymbol{R}\right) .
\end{aligned}
$$

Thus, $\mathrm{cl}_{\mathcal{T}_{\boldsymbol{P}}} \boldsymbol{R}=\mathrm{cl}_{\mathcal{T}_{\bar{P}}} \boldsymbol{R} \subseteq(\boldsymbol{P} \cap \overline{\boldsymbol{P}}) \cup\left(\boldsymbol{P} \cap \mathrm{cl}_{\mathcal{T}} \boldsymbol{R}\right)$. 
Following example shows that, in general

$$
\operatorname{cl}_{\mathcal{T}_{P}} \boldsymbol{R} \neq(\boldsymbol{P} \cap \overline{\boldsymbol{P}}) \cup\left(\boldsymbol{P} \cap \mathrm{cl}_{\mathcal{T}} \boldsymbol{R}\right) .
$$

Example 6.8. Let $X=[0,1]$. Let $C=\left(C_{L}, C_{U}\right)$ and $D=$ $\left(D_{L}, D_{U}\right)$ be the fuzzy rough sets of $X$, where

$$
\begin{aligned}
& C_{L}(x)=\left\{\begin{array}{l}
0.7, \text { if } x \neq 1, \\
0.0, \text { if } x=1,
\end{array} \quad C_{U}(x)=\left\{\begin{array}{l}
0.7, \text { if } x \neq 1, \\
0.5, \text { if } x=1,
\end{array}\right.\right. \\
& D_{L}(x)=\left\{\begin{array}{l}
0.3, \text { if } x \neq 1, \\
0.0, \text { if } x=1,
\end{array} \quad D_{U}(x)=\left\{\begin{array}{l}
0.3, \text { if } x \neq 1, \\
0.3, \text { if } x=1 .
\end{array}\right.\right.
\end{aligned}
$$

Then, $\boldsymbol{Q}=(C, D)$ is an IF rough set of $X$. Let $\mathcal{T}=\{\mathbf{0}, \mathbf{1}, \boldsymbol{Q}\}$. Thus, $(X, \mathcal{T})$ is a topological space of IF rough sets.

Let $A=\left(A_{L}, A_{U}\right)$ and $B=\left(B_{L}, B_{U}\right)$ be the fuzzy rough sets of $X$, where

$$
\begin{aligned}
& A_{L}(x)=\left\{\begin{array}{l}
0.5, \text { if } x \neq 1, \\
0.5, \text { if } x=1,
\end{array} \quad A_{U}(x)=\left\{\begin{array}{l}
0.6, \text { if } x \neq 1, \\
0.6, \text { if } x=1,
\end{array}\right.\right. \\
& B_{L}(x)=\left\{\begin{array}{l}
0.4, \text { if } x \neq 1, \\
0.0, \text { if } x=1,
\end{array} \quad B_{U}(x)=\left\{\begin{array}{l}
0.4, \text { if } x \neq 1, \\
0.4, \text { if } x=1 .
\end{array}\right.\right.
\end{aligned}
$$

Then, $\boldsymbol{P}=(A, B)$ is an IF rough set of $X$. Define $\mathcal{T}_{1}=\{\boldsymbol{P} \cap \boldsymbol{S} \mid$ $\boldsymbol{S} \in \mathcal{T}\}$. Then, $\mathcal{T}_{1}$ is a first topology on $\boldsymbol{P}$.

Let $M=\left(M_{L}, M_{U}\right)$ and $N=\left(N_{L}, N_{U}\right)$ be the fuzzy rough sets of $X$, where

$$
\begin{aligned}
& M_{L}(x)=\left\{\begin{array}{l}
0.4, \text { if } x \neq 1, \\
0.0, \text { if } x=1,
\end{array} \quad M_{U}(x)=\left\{\begin{array}{l}
0.4, \text { if } x \neq 1, \\
0.4, \text { if } x=1,
\end{array}\right.\right. \\
& N_{L}(x)=\left\{\begin{array}{l}
0.6, \text { if } x \neq 1, \\
0.0, \text { if } x=1,
\end{array} \quad N_{U}(x)=\left\{\begin{array}{l}
0.6, \text { if } x \neq 1, \\
0.5, \text { if } x=1 .
\end{array}\right.\right.
\end{aligned}
$$

Then, $\boldsymbol{R}=(M, N)$ is an IF rough set of $X$ such that $\boldsymbol{R} \subseteq \boldsymbol{P}$.

$$
\begin{gathered}
\text { Now, } \left.\operatorname{cl}_{\mathcal{T}_{1}} \boldsymbol{R}=\boldsymbol{P} \cap(\bigcap \overline{(\boldsymbol{P} \cap \boldsymbol{G})} \mid \overline{(\boldsymbol{P} \cap \boldsymbol{G})} \supseteq \boldsymbol{R} ; \boldsymbol{G} \in \mathcal{T}\}\right) \\
=\boldsymbol{P} \cap(\bigcap\{\overline{\boldsymbol{P}} \cup \overline{\boldsymbol{G}} \mid \overline{\boldsymbol{P}} \cup \overline{\boldsymbol{G}} \supseteq \boldsymbol{R}, \boldsymbol{G} \in \mathcal{T}\}) . \\
\overline{\boldsymbol{P}} \cup \overline{\mathbf{0}}=\overline{\boldsymbol{P}} \cup \mathbf{1}=\mathbf{1} \supseteq \boldsymbol{R}, \\
\overline{\boldsymbol{P}} \cup \overline{\mathbf{1}}=\overline{\boldsymbol{P}} \cup \mathbf{0}=\overline{\boldsymbol{P}} \nsupseteq \boldsymbol{R},
\end{gathered}
$$

because $A \nsubseteq N$. We then have $\overline{\boldsymbol{P}} \cup \overline{\boldsymbol{Q}}=(B \cup D, A \cap C) \supseteq \boldsymbol{R}$. Thus, $\mathrm{cl}_{\mathcal{T}_{1}} \boldsymbol{R}=\boldsymbol{P} \cap(\mathbf{1} \cap(\overline{\boldsymbol{P}} \cup \overline{\boldsymbol{Q}}))=\boldsymbol{P} \cap(\overline{\boldsymbol{P}} \cup \overline{\boldsymbol{Q}})$. If we write this set as $(F, G)$, then

$$
\begin{gathered}
F_{L}(x)=\left\{\begin{array}{l}
0.4, \text { if } x \neq 1, \\
0.0, \text { if } x=1,
\end{array} \quad F_{U}(x)=\left\{\begin{array}{l}
0.4, \text { if } x \neq 1, \\
0.4, \text { if } x=1,
\end{array}\right.\right. \\
G_{L}(x)=\left\{\begin{array}{l}
0.5, \text { if } x \neq 1, \\
0.0, \text { if } x=1,
\end{array} G_{U}(x)=\left\{\begin{array}{l}
0.6, \text { if } x \neq 1, \\
0.5, \text { if } x=1 .
\end{array}\right.\right.
\end{gathered}
$$

We have $\mathrm{cl}_{\mathcal{T}} \boldsymbol{R}=\bigcap\{\overline{\boldsymbol{T}} \mid \overline{\boldsymbol{T}} \supseteq \boldsymbol{R}, \boldsymbol{T} \in \mathcal{T}\}$. Thus, $\overline{\mathbf{0}}=\mathbf{1} \supseteq$ $\boldsymbol{R}, \overline{\mathbf{1}}=\mathbf{0} \nsupseteq \boldsymbol{R}, \overline{\boldsymbol{Q}} \nsupseteq \boldsymbol{R}$, because $C \nsubseteq N$. Thus, $\operatorname{cl}_{\mathcal{T}} \boldsymbol{R}=\mathbf{1}$. Therefore,

$$
\begin{aligned}
(\boldsymbol{P} \cap \overline{\boldsymbol{P}}) \cup\left(\boldsymbol{P} \cap \mathrm{cl}_{\tau} \boldsymbol{R}\right) & =(\boldsymbol{P} \cap \overline{\boldsymbol{P}}) \cup(\boldsymbol{P} \cap \mathbf{1}) \\
& =(\boldsymbol{P} \cap \overline{\boldsymbol{P}}) \cup \boldsymbol{P}=\boldsymbol{P}=(A, B) .
\end{aligned}
$$

Clearly, $(F, G) \subseteq(A, B)$ and $(F, G) \neq(A, B)$. Thus, cl $\mathcal{T}_{1} \boldsymbol{R}$ is a proper subset of $(\boldsymbol{P} \cap \overline{\boldsymbol{P}}) \cup\left(\boldsymbol{P} \cap \mathrm{cl}_{\mathcal{T}} \boldsymbol{R}\right)$.

\section{Conclusion}

In this paper, we introduced a topology for the redefined intuitionistic fuzzy rough sets and investigated the basic properties of their subspaces and continuous functions. We also introduced the concepts of the first and second transition spaces. We confirmed that these two spaces are different but share the same closed sets. In addition, we obtained the adjointness between the categories of fuzzy rough sets and intuitionistic fuzzy rough sets. All results obtained from this new definition are different from those of previous studies. The new approach of intuitionistic fuzzy rough sets enables us to manipulate them more simply and easily. In the following study, we will focus on expanding the related theories.

\section{Conflict of Interest}

No potential conflict of interest relevant to this article was reported.

\section{References}

[1] L. A. Zadeh, "Fuzzy sets," Information and Control, vol. 8, no. 3, pp. 338-353, 1965. https://doi.org/10.1016/S00199958(65)90241-X

[2] K. T. Atanassov, "Intuitionistic fuzzy sets," Fuzzy Sets and Systems, vol. 20, no. 1, pp. 87-96, 1986. https://doi. org/10.1016/S0165-0114(86)80034-3 
[3] Z. Pawlak, "Rough sets," International Journal of Computer \& Information Sciences, vol. 11, no. 5, pp. 341-356, 1982. https://doi.org/10.1007/BF01001956

[4] L. Zhou, W. Z. Wu, and W. X. Zhang, "On intuitionistic fuzzy rough sets and their topological structures," International Journal of General Systems, vol. 38, no. 6, pp. 589616, 2009. https://doi.org/10.1080/03081070802187723

[5] L. Zhou, W. Z. Wu, and W. X. Zhang, "On characterization of intuitionistic fuzzy rough sets based on intuitionistic fuzzy implicators," Information Sciences, vol. 179, no. 7, pp. 883-898, 2009. https://doi.org/10.1016/j.ins.2008.11. 015

[6] X. Yang and Y. Yang, "Independence of axiom sets on intuitionistic fuzzy rough approximation operators," International Journal of Machine Learning and Cybernetics, vol. 4, no. 5, pp. 505-513, 2013. https://doi.org/10.1007/ s13042-012-0116-6

[7] S. M. Yun and S. J. Lee, "Intuitionistic fuzzy rough approximation operators," International Journal of Fuzzy Logic and Intelligent Systems, vol. 15, no. 3, pp. 208-215, 2015. https://doi.org/10.5391/IJFIS.2015.15.3.208

[8] S. M. Yun and S. J. Lee, "Intuitionistic fuzzy topologies induced by intuitionistic fuzzy approximation spaces," International Journal of Fuzzy Systems, vol. 19, no. 2, pp. 285291, 2017. https://doi.org/10.1007/s40815-016-0237-2

[9] D. Dubois and H. Prade, "Rough fuzzy sets and fuzzy rough sets," International Journal of General System, vol. 17, no. 2-3, pp. 191-209, 1990. https://doi.org/10.1080/ 03081079008935107

[10] S. Nanda and S. Majumdar, "Fuzzy rough sets," Fuzzy Sets and Systems, vol. 45, no. 2, pp. 157-160, 1990. https: //doi.org/10.1016/0165-0114(92)90114-J

[11] D. Coker, "Fuzzy rough sets are intuitionistic L-fuzzy sets," Fuzzy Sets and Systems, vol. 96, no. 3, pp. 381-383, 1998. https://doi.org/10.1016/S0165-0114(97)00249-2

[12] K. Chakrabarty, R. Biswas, and S. Nanda, "Fuzziness in rough sets," Fuzzy Sets and Systems, vol. 110, no. 2, pp. 247-251, 2000. https://doi.org/10.1016/S0165-0114(97) 00414-4

[13] S. K. Samanta and T. K. Mondal, "Intuitionistic fuzzy rough sets and rough intuitionistic fuzzy sets," Journal of Fuzzy Mathematics, vol. 9, no. 3, pp. 561-582, 2001.
[14] W. Z. Wu and L. Zhou, "Topological structures of intuitionistic fuzzy rough sets," in Proceedings of 2008 International Conference on Machine Learning and Cybernetics, Kunming, China, 2008, pp. 618-623. https: //doi.org/10.1109/ICMLC.2008.4620478

[15] H. Hazra, S. K. Samanta, and K. C. Chattopadhyay, "Topology of intuitionistic fuzzy rough sets," Scientia Magna, vol. 7, no. 3, pp. 18-31, 2011.

[16] Z. Bashir, M. G. Abbas Malik, S. Asif, and T. Rashid, "The topological properties of intuitionistic fuzzy rough sets," Journal of Intelligent \& Fuzzy Systems, vol. 38, no. 1, pp. 795-807, 2020. https://doi.org/10.3233/JIFS179449

[17] J. Zhan and B. Sun, "Covering-based intuitionistic fuzzy rough sets and applications in multi-attribute decisionmaking," Artificial Intelligence Review, vol. 53, no. 1, pp. 671-701, 2020. https://doi.org/10.1007/s10462-018-96747

[18] S. A. Shanthi, "Application of intuitionistic fuzzy rough matrices," Advances and Applications in Mathematical Sciences, vol. 20, no. 4, pp. 501-513, 2021.

[19] S. M. Yun and S. J. Lee, "New approach to intuitionistic fuzzy rough sets," International Journal of Fuzzy Logic and Intelligent Systems, vol. 20, no. 2, pp. 129-137, 2020. https://doi.org/10.5391/IJFIS.2020.20.2.129

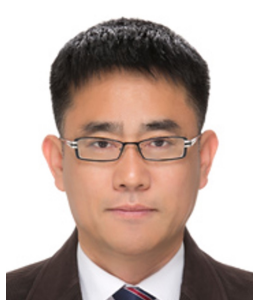

Sang Min Yun received his Ph.D. degree from Chungbuk National University in 2015. He is a lecturer at the Department of Mathematics, Chungbuk National University since 2015 . His research interests include general topology and fuzzy topology. He is a member of KIIS and KMS.

E-mail: jivesm@naver.com

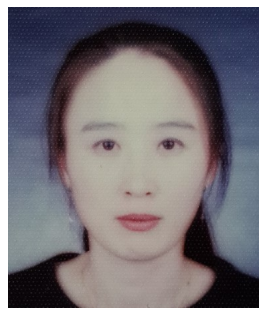

Yeon Seok Eom received her Ph.D. degree from Chungbuk National University in 2012. She is a lecturer at the Department of Mathematics, Chungbuk National University since 2012. Her research interests include general topology and fuzzy topology. She is a member of KIIS and

KMS.

E-mail: math1518@naver.com 


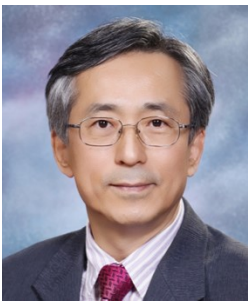

Seok Jong Lee received his M.S. and Ph.D. degrees from Yonsei University in 1986 and 1990, respectively. He is a professor at the Department of Mathematics, Chungbuk National University since 1989. He was a visiting scholar in Carleton University from 1995 to 1996, and Wayne State University from 2003 to 2004. His research interests include general topology and fuzzy topology. He is a member of KIIS and KMS.

E-mail: sjl@cbnu.ac.kr 